\title{
A Comprehensive Evaluation of the Relationship Between Fall Risk and Other Geriatric Syndromes
}

(D) Güzin Çakmak

Gaziantep University Faculty of Medicine, Department of Internal Medicine, Division of Geriatric Medicine, Gaziantep, Turkey

\begin{abstract}
Objective: Falls are important geriatric syndromes that can result in morbidity and mortality in elderly individuals. Many factors increase the risk of falls. To effectively control falls it is important to reveal its relationship with other geriatric syndromes.

Materials and Methods: This cross-sectional study was conducted for a period of 4 months from September 2019 to January 2020. Fall risk assessment was done with the Tinetti test and the timed up and go (TUG) test. All patients underwent comprehensive geriatric assessment tests.

Results: The study population was composed of 93 women and 57 men, and the mean age was $73 \pm 9.2$ years. It was observed that the scores obtained from the Katz and Lawton-Brody scales were lower in patients with a high risk of falls. Handgrip strength, skeletal muscle mass index, mininutritional assessment score, and mini-mental status assessment score were lower in these patients. Geriatric depression scale score, fried and SOF frailty index scores, and the number of drugs used were higher in them. Frailty, handgrip strength, gait speed, and depression were independently associated with Tinetti-measured fall risk in linear regression analysis $\left(R^{2}=0.414 ; p<0.001, p=0.004, p=0.002, p=0.027\right)$. IADL and gait speed were found to be independently associated with fall risk according to TUG $\left(R^{2}=0.550 ; p=0.012, p<0.001\right)$.
\end{abstract}

Conclusion: In our study, we concluded that there is a close relationship between the risk of falls and other geriatric syndromes. Effective management of geriatric syndromes will help reduce the risk of falls.

Keywords: Fall, geriatric syndromes, timed up and go, Tinetti

\section{Introduction}

Falls are important problems that limit independent mobility in older adults and are considered as one of the important geriatric syndromes (1). It is known that falls cause an increase in hospital stays and health expenditures due to complications such as injuries and fractures (2). About 30\% of adults aged 65 and over have been experiencing falls each year (3). The causes of falls can be examined under two main headings as intrinsic and extrinsic causes. We can consider intrinsic factors in the two groups as age-related physiological and pathological changes. The decrease in visual sensitivity, age-related hearing loss, decrease in tactile sense and vibration sense, and balance disorders can be considered as examples of age-related physiological changes. An increase in fat mass and decrease in muscle mass with age can also be listed among them. Stroke, dementia, parkinsonism, epilepsy, and carotid sinus hypersensitivity syndrome are among the pathological causes of falls (4).

Conditions that are more common in old age and known to reduce life expectancy, such as dementia, depression, delirium, incontinence, falls, frailty, sarcopenia, and malnutrition, are called geriatric syndromes (5). Geriatric syndromes are usually seen together, not as isolated clinical conditions. Co-occurrence of geriatric syndromes can be explained by the fact that they have a common pathogenesis. They can be prevented and treated with similar approaches.

It is important to reveal the relationship between falls and other geriatric syndromes to determine preventive and therapeutic approaches. Although studies are describing these relationships separately, there is no study evaluating the relationship between fall risk and all geriatric syndromes together. We aimed to

Address for Correspondence: Güzin Çakmak, Gaziantep University Faculty of Medicine, Department of Internal Medicine, Division of Geriatric Medicine, Gaziantep, Turkey

Phone: +90 3423416689 E-mail: drguzincakmak@gmail.com ORCID: orcid.org/0000-0002-1046-690X

Received: 28.09.2021 Accepted: 28.12.2021

Cite this article as: Çakmak G. A Comprehensive Evaluation of the Relationship Between Fall Risk and Other Geriatric Syndromes.

Eur J Geriatr Gerontol 2022;4(2):58-63

๑Copyright 2022 by the Academic Geriatrics Society / European Journal of Geriatrics and Gerontology published by Galenos Publishing House. 
examine the relationship between fall risk and other geriatric syndromes in this study.

\section{Materials and Methods}

\section{Participants}

This cross-sectional study was carried out for a period of 4 months from September 2019 to January 2020. Patients who applied to the geriatrics outpatient clinic were included in the study. The Local Research Ethics Committee approved the study (number: 2019/269, date: 03.07.2019). All participants gave informed consent.

\section{Exclusion criteria}

Patients, who were below 65 years of age, and had already been diagnosed with any of the geriatric syndromes such as sarcopenia, frailty, malnutrition, polypharmacy, fall, urinary and fecal incontinence were not included in the study. Those with cancer and severe inflammatory disease were also excluded from the study.

\section{Assessment of fall risk}

Tinetti balance-gait evaluation scale and timed up and go test (TUG) were used for evaluation of the fall risk. Tinetti assessment tool scores gait and balance according to the ability to perform certain tasks. In the Tinetti balance-gait evaluation scale, a score of $>24$ means low risk of fall, 19-23 moderate risk of fall, and $<19$ high risks of fall (6). TUG is a test that evaluates both static and dynamic balance. It uses the time it takes for the person to get up from the chair, walk three meters, turn 180 degrees, walk back to the chair, and sit. Patients who took 14 seconds, or longer from the TUG test, were classified as high-risk for fall too (7).

\section{Comprehensive geriatric assessment}

A cognitive evaluation was done by the standardized form of mini-mental state examination (MMSE), assessment of abilities of daily living (ADL) by Katz index, and instrumental activities of daily living (IADL) by the Lawton Brody index. The evaluation of psychological status was done by the short form of the Yesavage geriatric depression scale.

In MMSE, patients were evaluated for six different areas; orientation, attention, registration, language, calculation, and recall (8). Patients whose scores $\leq 24$ were evaluated as dementia (9). Patients were evaluated for personal hygiene, continence, toileting, dressing, feeding, and ambulating by Katz index of ADL. High scores were considered high self-sufficiency (10). Lawton Brody index was used for evaluating IADL like house cleaning, doing the laundry, shopping, managing medications, cooking, communicating with others, using transportation, and doing financial management; higher scores meant higher independence (11). Patients with GDS scores of 5 and higher were accepted as positive for depression (12).

Malnutrition was assessed by a mini nutritional assessment, short-formed (MNA-SF), long-form (MNA-LF), and the Global Leadership Initiative on malnutrition criteria (GLIM) (13-15). MNA-SF is the first part of the MNA test and takes only a few minutes to complete. The maximum score for MNA-SF is 14; a score of 12 points or greater indicates that the patient has an acceptable nutritional status and the full MNA is not needed to be done. However, a score of 11 points or below is an indication to proceed with the MNA-LF. Completing the MNA-LF takes 10 to 15 minutes. The maximum score for the second part is 16 and the maximum score for MNA-LF is 30 . Scores $<17$ are accepted as malnutrition, 17-23.5 is accepted as the risk for malnutrition, and 24-30 is accepted as normal. GLIM criteria are new criteria for diagnosing malnutrition. These criteria are based on the verification of phenotypic criteria, such as low body mass index, non-volitional loss of weight, or reduced muscle mass, associated with etiological variables, such as reduced food intake, the presence of inflammation, or disease burden. Also, the stage of malnutrition can be determined as moderate and severe according to body mass index, loss of weight, and muscle mass.

\section{Assessment for sarcopenia}

For defining sarcopenia, muscle strength, muscle mass, and physical performance were assessed. SARC-F (strength, assistance need for walking, rising from a chair, climbing stairs, and falls) test was used to select cases to be evaluated for muscle strength (16). The handgrip test was performed if the patient had a score $\geq 4$ from SARC- $F$ to diagnose probable sarcopenia. The handgrip test was performed by using a hand dynamometer with the dominant hand. For females $<16 \mathrm{~kg}$ (kilograms), for males $<27$ $\mathrm{kg}$ were accepted as probable sarcopenic (17). A bioimpedance test was carried out on probable sarcopenic patients to assess skeletal muscle mass. Sarcopenia was diagnosed by skeletal muscle mass index. In this study, we used skeletal muscle mass index (SMMI) adjusted to height. SMMI was calculated by dividing skeletal muscle mass by the square of height (18). We evaluated gait speed with a four-meter gait speed test to diagnose severe sarcopenia (19).

\section{Assessment for frailty syndrome}

Frailty was assessed by using the fried frailty index (FFI) and study of osteoporotic fractures (SOF) index. FFI is constituted from five criteria: Unintentional weight loss, self-reported poor energy, weakness (reduced grip strength), slow gait speed, and low physical activity. People who were positive for three and more FFI criteria were defined as frail. Those who were positive for one or two criteria were described as pre-frail (20). The SOF index evaluates frailty syndrome by using three components 
(weight loss, inability to rise from a chair five times without using the arms, and reduced energy). The presence of frailty syndrome was determined by the presence of two or more of the three criteria in the SOF index. Patients that were positive for 1 criterion were described as pre-frail (21). People not meeting any criteria were defined as robust in both assessment tools.

\section{Statistics}

The variables were analyzed for the normality of their distribution using the Kolmogorov-Smirnov test. All data were normally distributed. Numerical variables were denoted as mean \pm standard deviation. Categorical variables were represented as frequencies. Comparison groups were done by independent sample t-test and ANOVA. A univariate linear regression model was used to study the linear relationship between the risk of falls and other geriatric syndromes. The significance check in the linear regression analysis was based on an F-test and the significance of single independent variables was assessed by a t-test. The IBM SPSS for Windows, version 22.0 (IBM Corp., Armonk, NY, USA) was used for statistical analysis.

\section{Results}

One hundred and fifty subjects were included in this study evaluating the relationship between fall risk and geriatric syndromes. Fifty-six were women and 94 were men. The mean age was $73 \pm 9.2$. ADL and IADL seemed to be impaired more in patients with a high risk of fall than in patients with low risk when evaluated with the Tinetti test $(p<0.001, p=0.044)$. Nutritional status evaluated by MNA, MNA-SF, and GLIM criteria were found to be better in patients with a low risk of fall than in patients with moderate and high risk $(p<0.001)$. Handgrip strength was preserved better in those with a low risk than in those with a high risk $(p=0.003)$. Gait speed was also higher in those who were at low risk of fall when compared to the patients who had moderate and high risk $(p<0.001)$. It was observed that low-risk patients met the criteria of fried and SOF frailty indexes less than others $(p<0.001)$. The geriatric depression score was also lower in those with a low risk of fall compared to those with a moderate and high risk of fall $(p=0.008, p<0.001)$. The number of drugs used was higher in high-risk patients than in low-risk patients $(p=0.003)$. The relationship between fall risk and other geriatric syndromes according to the Tinetti test is summarized in Table 1.

When we evaluated the fall risk with TUG, we found that ADL and IADL were better in those with a low risk of fall $(p<0.001)$. The MMSE score was lower in those with a high risk of falls $(p=0.003)$. Fried and SOF frailty index scores were higher in those with a high risk of fall $(p=0.003, p=0.001)$. Handgrip strength, SMMI, and gait speed were decreased in the high-risk group ( $p=0.023, p<0.001, p<0.001)$. The number of drugs used was higher in the high-risk group $(p=0.032)$. The relationship between fall risk and other geriatric syndromes according to the TUG test is summarized in Table 2.

We found that the FFI score, handgrip strength, gait speed, and GDS were independently related to fall risk measured with Tinetti in linear regression analysis $\left(R^{2}=0.414 ; p<0.001, p=0.004\right.$, $p=0.002, p=0.027)$. IADL and gait speed were independently related to fall risk according to the TUG in linear regression analysis $\left(R^{2}=0.550 ; p=0.012, p<0.001\right)$. In the linear regression analysis performed with the risk of falling according to the Tinetti test, the R2 value was 40\% in the analysis and 50\% in the analysis performed with the TUG. Considering this, we can say that the model fit is moderate. Results of linear regression analysis were summarized in Tables 3 and 4.

\section{Discussion}

In our study, we observed that the risk of falls was associated with ADL and IADL. Sekaran et al. (22) had evaluated the association between falls and ADL in a 10-year prospective cohort study in adults aged 65-69 years. The researchers concluded that there is a significant association between increased risk of falls and ADL difficulties (22). Brown et al. (23) revealed that loss of IADL was strongly associated with the risk of falls. The authors suggested that IADL levels could be a powerful and practical tool for screening patients for fall risk (23). Because the risk of fall makes independent movement more difficult, the risk of fall and disability are closely related.

We found a significant relationship between the risk of falls and malnutrition. Bórikova et al. (24) had shown the association between malnutrition and the risk of fall, in their study. The authors revealed that none of the patients with a history of a single fall were suffered from malnutrition at hospitalization, but malnutrition was frequently seen in those with a history of multiple falls (24). Malnutrition can lead to nutrient deficiencies such as vitamin B12 deficiency, leading to loss of balance and fall. In addition, muscle wasting, which is directly affected by malnutrition, can increase the risk of falls. Frailty was also related to fall risk according to our study results. Cognitive and physical fragility, which are components of frailty, can increase the risk of falls (25). Clegg et al. (26) have previously shown that frailty is associated with an increased risk of falls Additionally, more than half of the frail adults suffered from falls.

In our study, high GDS scores and low MMSE were found to be related to falling risk. Kamińska et al. (27) also revealed that the risk of fall is associated with regression in $A D L$, depression, and cognitive decline. Unlike our study, cognitive functions were evaluated with abbreviated mental test scores in this study. The relationship between depression, dementia, and the risk of falls can be attributed to many reasons. Both situations are accompanied by distraction, we can specify this as one of the reasons. In both cases, antipsychotics that have a place in the 


\begin{tabular}{|c|c|c|c|c|}
\hline & $\begin{array}{l}\text { Low-risk } \\
(n=109) \\
(\text { mean } \pm \text { SD) }\end{array}$ & $\begin{array}{l}\text { Moderate-risk } \\
(n=21) \\
(\text { mean } \pm \text { SD) }\end{array}$ & $\begin{array}{l}\text { High-risk } \\
(n=20) \\
(\text { mean } \pm \text { SD) }\end{array}$ & $\mathbf{p}$ \\
\hline ADL & $5.4 \pm 0.9$ & $5.1 \pm 1$ & $4.3 \pm 1.7$ & $<0.001^{*}$ \\
\hline IADL & $5.4 \pm 2.9$ & $3.9 \pm 2.2$ & $4 \pm 2.2$ & $0.044^{*}$ \\
\hline MNA & $23.3 \pm 4$ & $20.3 \pm 4.9$ & $19.6 \pm 3.8$ & $<0.001^{*}$ \\
\hline MNA-SF & $11.5 \pm 3.5$ & $8.4 \pm 2.4$ & $8.4 \pm 2.1$ & $<0.001^{*}$ \\
\hline GLIM (\% of malnourished) & $11.9 \%$ & $42.9 \%$ & $65 \%$ & $<0.001^{*}$ \\
\hline $\mathrm{FFI}$ & $2 \pm 1.2$ & $3.3 \pm 1.3$ & $4.3 \pm 0.7$ & $<0.001^{*}$ \\
\hline SOF & $1.1 \pm 0.9$ & $1.9 \pm 1$ & $2.3 \pm 0.6$ & $<0.001^{*}$ \\
\hline Handgrip strength (kg) & $30.5 \pm 15.5$ & $26.2 \pm 11.1$ & $20.2 \pm 7.1$ & $0.003^{*}$ \\
\hline SMMI $\left(\mathrm{kg} / \mathrm{m}^{2}\right)$ & $11 \pm 1.6$ & $10.9 \pm 1.4$ & $11.1 \pm 1.5$ & 0.91 \\
\hline Gait speed (m/s) & $0.6 \pm 0.1$ & $0.4 \pm 0.1$ & $0.4 \pm 0.2$ & $<0.001^{*}$ \\
\hline GDS & $9.3 \pm 5.6$ & $13.1 \pm 6.9$ & $17.1 \pm 6.2$ & $<0.001^{*}$ \\
\hline Number of drugs used & $6.2 \pm 3$ & $7.6 \pm 4.3$ & $8.6 \pm 3.1$ & $0.003^{*}$ \\
\hline \multicolumn{5}{|c|}{$\begin{array}{l}\text { ADL: Activities of daily living, IADL: Instrumental activities of daily living, MNA: Mini nutritional assessment, MNA-SF: Mini nutritional assessment-short form, GLIM: Global leadership } \\
\text { initiative on malnutrition, FFI: Fried frailty index, SOF: Study of osteoporotic fractures, SMMI: Skeletal muscle mass index, GDS: Geriatric depression scale, kg: Kilograms, kg/m²: Kilogram } \\
\text { per square meter, m/s: Meter per second, SD: Standard deviation }\end{array}$} \\
\hline
\end{tabular}

\begin{tabular}{|c|c|c|c|}
\hline & $\begin{array}{l}\text { Low-risk } \\
(n=57) \\
(\text { mean } \pm S D)\end{array}$ & $\begin{array}{l}\text { High-risk } \\
(n=93) \\
(\text { mean } \pm \text { SD) }\end{array}$ & $\mathbf{p}$ \\
\hline ADL & $5.7 \pm 0.5$ & $5 \pm 1.3$ & $<0.001^{*}$ \\
\hline IADL & $6 \pm 2.5$ & $4.3 \pm 2.8$ & $<0.001^{*}$ \\
\hline $\mathrm{FFI}$ & $2.1 \pm 1.5$ & $2.9 \pm 1.4$ & $<0.001^{*}$ \\
\hline SOF & $1.04 \pm 0.8$ & $1.6 \pm 1$ & $<0.001^{*}$ \\
\hline MMSE & $24 \pm 5.5$ & $20.7 \pm 7.4$ & $0.003^{*}$ \\
\hline Handgrip strength $(\mathrm{kg})$ & $30.7 \pm 13.3$ & $25.2 \pm 12.9$ & $0.023^{*}$ \\
\hline SMMI $\left(\mathrm{kg} / \mathrm{m}^{2}\right)$ & $11.6 \pm 1$ & $10.6 \pm 1.7$ & $<0.001^{*}$ \\
\hline Gait speed $(\mathrm{m} / \mathrm{s})$ & $0.6 \pm 0.07$ & $0.4 \pm 0.1$ & $<0.001^{*}$ \\
\hline Number of drugs used & $6.1 \pm 3.1$ & $7.3 \pm 3.2$ & $0.032^{*}$ \\
\hline \multicolumn{4}{|c|}{$\begin{array}{l}\text { ADL: Activities of daily living, IADL: Instrumental activities of daily living, FFI: Fried } \\
\text { frailty index, SOF: Study of osteoporotic fractures, SMMI: Skeletal muscle mass index, } \\
\text { GDS: Geriatric depression scale, } \mathrm{kg} / \mathrm{m}^{2} \text { : Kilogram per square meter, } \mathrm{m} / \mathrm{s} \text { : Meter per } \\
\text { second, SD: Standard deviation }\end{array}$} \\
\hline
\end{tabular}

treatment may also increase the risk of falls in older individuals. In both cases, deterioration of nutritional status can lead to muscle loss and falls.

We revealed that the risk of fall was found to be associated with the parameters evaluating sarcopenia. Different studies have shown that sarcopenia is one of the parameters that increase the risk of falls in older adults. These studies were reviewed in a metaanalysis by Yeung et al. (28). The positive association between sarcopenia and falls in older adults reinforces the need to take measures to see the impact of sarcopenia prevention on falls.

We also concluded that the increase in the number of drugs used increases the risk of falls. In the study of Dhalwani et al. (29), the use of $\geq 4$ drugs increased the frequency of falls by $18 \%$, while the use of $\geq 10$ drugs increased the frequency of falls by $50 \%$. The relationship between the increase in the number of drugs used and the increase in the risk of fall may be related to the use of drugs that increase the risk of fall. According to the study of Woolcott et al. (30) antidepressants, neuroleptics, antipsychotics, benzodiazepines, sedative-hypnotics, and antihypertensive drugs were shown as the drugs that increase the risk of falling the most. In addition, it should be taken into account that people with multimorbidity will use drugs more and these people may have co-morbid diseases that may trigger falls.

In their article, van der Velde and Seppala (31) stated that there is a lack of knowledge about the role of drugs as a fall risk factor in caregivers of the elderly. They also stated that the members of the task $\&$ finish group of European Geriatric Medicine Association are engaged in activities aimed at raising public awareness and disseminating information to facilitate appropriate (de)prescription in elderly people at risk of fall. It is important to increase such educational activities for healthcare professionals, caregivers, and patients about drugs that increase the risk of falls (31).

Measures that can be taken to prevent falls are not limited to deprescribing risky drugs. Exercises aimed at strengthening muscles and increasing balance are among them too. Correction of visual and hearing disorders, improvement of nutrition, and support of cognitive functions are other measures that can be taken. It is also important to make the environmental conditions safe for the patients at risk of falls (32).

Although there have been studies evaluating the relationship between fall risks and different geriatric syndromes, there is no 


\begin{tabular}{|c|c|c|c|c|c|}
\hline Independent variables & B & St. error & Beta & $\mathbf{T}$ & p \\
\hline $\mathrm{ADL}$ & -0.053 & 0.076 & -0.069 & -0.696 & 0.488 \\
\hline Handgrip strength & 0.001 & 0.005 & 0.018 & 0.212 & $0.004^{*}$ \\
\hline SMMI & 0.111 & 0.037 & 0.235 & 2.961 & 0.833 \\
\hline SOF & 0.022 & 0.091 & 0.030 & 0.243 & 0.808 \\
\hline MNA & -0.005 & 0.017 & -0.030 & -0.241 & 0.771 \\
\hline MNA-SF & 0.005 & 0.020 & 0.023 & 0.237 & 0.813 \\
\hline GLIM & 0.007 & 0.103 & 0.008 & 0.069 & 0.945 \\
\hline
\end{tabular}

Table 4. Results of linear regression analysis

\begin{tabular}{|c|c|c|c|c|c|}
\hline Independent variables & B & St. error & Beta & $\mathbf{T}$ & $\mathbf{p}$ \\
\hline$A D L$ & 0.019 & 0.036 & 0.042 & 0.527 & 0.559 \\
\hline IADL & -0.045 & 0.018 & -0.258 & -2.537 & $0.012^{*}$ \\
\hline $\mathrm{FFI}$ & 0.011 & 0.033 & 0.033 & 0.334 & 0.739 \\
\hline SOF & -0.038 & 0.049 & -0.076 & -0.786 & 0.434 \\
\hline Number of drugs used & 0.000 & 0.010 & 0.001 & 0.014 & 0.989 \\
\hline
\end{tabular}

study evaluating the effect of many geriatric syndromes on fall risk at the same time. This is a strong side of our work.

\section{Study Limitations}

The most important limitation of our study is that multiple analyzes were performed with data from a relatively small sample. In a prospective study with a larger sample, the relationship between geriatric syndromes and falls, fall-related morbidity, and mortality can be revealed. Another limitation of the study is that the assessment of depression made by the geriatric depression scale was not confirmed by the clinical assessment made by a psychiatrist. The relationship between depression and fall can be better demonstrated with a prospective study in which both detailed psychiatric evaluation and response to depression treatment and drug side effects are evaluated.

\section{Conclusion}

In our study, it was concluded that disability, malnutrition, frailty, sarcopenia, depression, and polypharmacy were more common in patients with a high risk of falls. In addition, deterioration in cognitive functions was also higher.
Falls are important geriatric syndromes that can result in morbidity and mortality in the elderly. Predicting situations that increase the risk of falling is important for effective planning of the measures to be taken. Revealing the relationship with geriatric syndromes leads us to the conclusion that effective management of geriatric syndromes can reduce the risk of falls. It would be useful to carry out studies evaluating the measures and effectiveness that can be taken in this regard.

\section{Ethics}

Ethics Committee Approval: The study protocol was approved by the Local Research Ethics Committee (number: 2019/269, date: 03.07.2019).

Informed Consent: All participants gave informed consent.

Peer-review: Externally peer-reviewed.

Financial Disclosure: The author declared that this study received no financial support.

\section{References}

1. Duque G, Kiel DP. Osteoporosis in older persons: Advances in pathophysiology and therapeutic approaches: 2th ed. Osteoporos Older Pers Adv Pathophysiol Ther Approaches Second Ed, 2016, pp. 1-282. 
2. Florence CS, Bergen G, Atherly A, Burns E, Stevens J, Drake C. The Medical Costs of Fatal Falls and Fall Injuries among Older Adults. J Am Geriartics Soc 2018;66:693-698.

3. Morrison A, Fan T, Sen SS, Weisenfluh L, Weisenfluh L. Epidemiology of falls and osteoporotic fractures: a systematic review. Clinicoecon Outcomes Res 2013;5:9-18.

4. Pasquetti $P$, Apicella $L$, Mangone G. Pathogenesis and treatment of falls in elderly. Clin Cases Miner Bone Metab 2014;11:222-225.

5. Liang $Y$, Rausch $C$, Laflamme L, Möller J. Prevalence, trend and contributing factors of geriatric syndromes among older Swedes: Results from the Stockholm County Council Public Health Surveys. BMC Geriatr 2018;18:322.

6. Chu LW, Chiu AYY, Chi I. Impact of falls on the balance, gait, and activities of daily living functioning in community-dwelling Chinese older adults. J Gerontol A Biol Sci Med Sci 2006;61:399-404.

7. Barry E, Galvin R, Keogh C, Horgan F, Fahey T. Is the Timed Up and Go test a useful predictor of risk of falls in community dwelling older adults: A systematic review and meta- analysis. BMC Geriatr 2014;14:14.

8. Mitchell AJ. A meta-analysis of the accuracy of the mini-mental state examination in the detection of dementia and mild cognitive impairment. J Psychiatr Res 2009;43:411-431.

9. Piotrowicz K, Romanik W, Skalska A, Gryglewska B, Szczerbińska K, Derejczyk J, Krzyżewski RM, Grodzicki T, Gąsowski J. The comparison of the 1972 Hodkinson's Abbreviated Mental Test Score (AMTS) and its variants in screening for cognitive impairment. Aging Clin Exp Res 2019;31:561-566.

10. Shelkey M, Wallace M. Katz Index of Independence in Activities of Daily Living (ADL). Director 2000;8:72-73.

11. Oort Q, Taphoorn MJB, Sikkes SAM, Uitdehaag BMJ, Reijneveld JC, Dirven L. Evaluation of the content coverage of questionnaires containing basic and instrumental activities of daily living (ADL) used in adult patients with brain tumors. J Neurooncol 2019;143:1-13.

12. Lin X, Haralambous B, Pachana NA, Bryant C, Logiudice D, Goh A, Dow B. Screening for depression and anxiety among older Chinese immigrants living in Western countries: The use of the Geriatric Depression Scale (GDS) and the Geriatric Anxiety Inventory (GAI). Asia Pac Psychiatry 2016;8:3243.

13. Kaiser MJ, Bauer JM, Ramsch C, Uter W, Guigoz $Y$, Cederholm T, Thomas DR, Anthony P, Charlton KE, Maggio M, Tsai AC, Grathwohl D, Vellas B, Sieber CC; MNA-International Group. Validation of the Mini Nutritional Assessment short-form (MNA-SF): A practical tool for identification of nutritional status. J Nutr Heal Aging 2009;13:782-788.

14. Bauer JM, Kaiser MJ, Anthony P, Guigoz Y, Sieber CC. The mini nutritional assessment ${ }^{\circledR}$-its history, today's practice, and future perspectives. Nutr Clin Pract 2008;23:388-396.

15. Cederholm T, Jensen GL, Correia MITD, Gonzalez MC, Fukushima $R$, Higashiguchi T, Baptista G, Barazzoni R, Blaauw R, Coats A, Crivelli A, Evans DC, Gramlich L, Fuchs-Tarlovsky V, Keller H, Llido L, Malone A, Mogensen KM, Morley JE, Muscaritoli M, Nyulasi I, Pirlich M, Pisprasert V, de van der Schueren MAE, Siltharm S, Singer P, Tappenden K, Velasco N, Waitzberg D, Yamwong P, Yu J, Van Gossum A, Compher C; GLIM Core Leadership Committee; GLIM Working Group. GLIM criteria for the diagnosis of malnutrition - A consensus report from the global clinical nutrition community. Clin Nutr 2019;38:1-9.

16. Cao L, Chen S, Zou C, Ding X, Gao L, Liao Z, Liu G, Malmstrom TK, Morley JE, Flaherty JH, An Y, Dong B. A pilot study of the SARC-F scale on screening sarcopenia and physical disability in the Chinese older people. J Nutr Heal Aging 2014;18:277-283.
17. Cruz-Jentoft $A J$, Bahat $G$, Bauer J, Boirie $Y$, Bruyère 0 , Cederholm T, Cooper C, Landi F, Rolland $Y$, Sayer AA, Schneider SM, Sieber CC, Topinkova E, Vandewoude $M$, Visser M, Zamboni M; Writing Group for the European Working Group on Sarcopenia in Older People 2 (EWGSOP2), and the Extended Group for EWGSOP2. Sarcopenia: Revised European consensus on definition and diagnosis. Age Ageing 2019;48:16-31.

18. Gąsior JS, Pawłowski M, Williams CA, Dąbrowski MJ, Rameckers EA. Assessment of maximal isometric hand grip strength in school-aged children. Open Med (Wars) 2018;13:22-28.

19. Bahat G, Tufan A, Kilic C, Aydın T, Akpinar TS, Kose M, Erten N, Karan MA, Cruz-Jentoft AJ. Cut-off points for height, weight and body mass index adjusted bioimpedance analysis measurements of muscle mass with use of different threshold definitions. Aging Male 2020;23:382-387.

20. Hoogendijk EO, Afilalo J, Ensrud KE, Kowal P, Onder G, Fried LP. Frailty: implications for clinical practice and public health. Lancet 2019;394:13651375.

21. De Buyser SL, Petrovic M, Taes YE, Toye KR, Kaufman JM, Lapauw B, Goemaere S. Validation of the FNIH sarcopenia criteria and SOF frailty index as predictors of long-term mortality in ambulatory older men. Age Ageing 2016;45:602-608.

22. Sekaran NK, Choi H, Hayward RA, Langa KM. Fall-associated difficulty with activities of daily living in functionally independent individuals aged 65 to 69 in the United States: a cohort study. J Am Geriatr Soc 2013;61:96-100.

23. Brown J, Kurichi JE, Xie D, Pan O, Stineman MG. Instrumental activities of daily living staging asa possible clinical tool for falls risk assessment in physical medicine and rehabilitation. PM R 2014;6:316-323.

24. Bóriková I, Tomagová M, Žiaková K, Miertová M. Pharmacotherapy as a fall risk factor. Cent Eur J Nurs Midwifery 2018;9:832-839.

25. Bandeen-Roche K, Seplaki CL, Huang J, Buta B, Kalyani RR, Varadhan R, Xue OL, Walston JD, Kasper JD. Frailty in Older Adults: A Nationally Representative Profile in the United States. J Gerontol Ser A Biol Sci Med Sci 2015;70:1427-1434.

26. Clegg A, Young J, lliffe S, Rikkert MO, Rockwood K. Frailty in elderly people. Lancet 2013;381:752-762.

27. Kamińska MS, Brodowski J, Karakiewicz B. Fall risk factors in communitydwelling elderly dependingon their physical function, cognitive status and symptoms of depression. Int J Environ Res Public Health 2015;12:34063416

28. Yeung SSY, Reijnierse EM, Pham VK, Trappenburg MC, Lim WK, Meskers CGM, Maier AB. Sarcopenia and its association with falls and fractures in older adults: A systematic review and meta-analysis. J Cachexia Sarcopenia Muscle 2019;10:485-500.

29. Dhalwani NN, Fahami R, Sathanapally H, Seidu S, Davies MJ, Khunti K. Association between polypharmacy and falls in older adults: A longitudinal study from England. BMJ Open 2017;7:e016358.

30. Woolcott JC, Richardson KJ, Wiens MO, Patel B, Marin J, Khan KM, Marra CA. Meta-analysis of the impact of 9 medication classes on falls in elderly persons. Arch Intern Med 2009;160:1952-1960.

31. van der Velde N, Seppala L. Can We STOPP Falls? Eur J Geriatr Gerontol 2021;3:32-34.

32. Blain H, Miot S, Bernard PL. How Can We Prevent Falls? In:Falaschi P, Marsh D, eds. Orthogeriatrics: The Management of Older Patients with Fragility Fractures. Cham (CH): Springer 2020, pp 273-90. 